\title{
Individual variability of olfactory fMRI in normosmia and olfactory dysfunction
}

\author{
Zang Yunpeng ${ }^{1,2}$ (D) Pengfei Han ${ }^{1,3} \cdot$ Akshita Joshi $^{1} \cdot$ Thomas Hummel $^{1}$
}

Received: 29 May 2020 / Accepted: 18 July 2020 / Published online: 14 August 2020

(c) The Author(s) 2020

\begin{abstract}
Purpose The diagnosis of olfactory dysfunction is mainly based on psychophysical measurements. The aim of the current study was to investigate how well the olfactory functional magnetic resonance imaging (fMRI) can effectively distinguish between normosmic people and subjects with olfactory dysfunction.

Methods Thirty-eight participants were recruited for the study. Group 1 consisted of 22 subjects with olfactory dysfunction (mean age $=44.3$ years, $\mathrm{SD}=18.6$ ), and Group two consisted of 16 participants with normal olfactory function (mean age $=49.6$ years, $\mathrm{SD}=11.6$ ). Olfactory functions were assessed in great detail for all participants, and brain activation in response to odorous stimulation was assessed using fMRI.

Results The between-group comparison showed stronger odor induced brain activation of the primary olfactory area and the insular cortex among the normosmic group as compared to the dysosmic group. As indicated by the individual analysis, positive responses in the primary olfactory cortex were significantly higher in normosmic people (94\%) than in subjects with olfactory dysfunction (41\%). However, there was no association between individual fMRI parameters (including the percentage of BOLD signal change, activated cluster size and peak $z$ value), and psychophysical olfactory test scores. Receiver operating characteristic analysis suggested the subjects could not be differentiated from normosmics based on their BOLD signal from the primary olfactory area, orbitofrontal cortex, or the insular cortex.

Conclusion There are large inter-individual variabilities for odor-induced brain activation among normosmic subjects and subjects with olfactory dysfunction, due to this variation, at present it appears problematic to diagnose olfactory dysfunction on an individual level using fMRI.
\end{abstract}

Keywords fMRI $\cdot$ Olfactory dysfunction $\cdot$ Individual variability $\cdot$ Objective diagnosis

Zang Yunpeng and Pengfei Han contributed equally to this work.

Zang Yunpeng

yunpengzang23@gmail.com

Pengfei Han

pengfeihan88@gmail.com

Akshita Joshi

joshiakshita93@gmail.com

Thomas Hummel

thummel@mail.zih.tu-dresden.de

1 Department of Otorhinolaryngology, Interdisciplinary Center Smell and Taste, Medical Faculty Carl Gustav Carus, TU Dresden, Fetscherstrasse 74, 01307 Dresden, Germany

2 Department of Otorhinolaryngology, The Affiliated Hospital XuZhou Medical University, XuZhou, People's Republic of China

3 Faculty of Psychology, Southwest University, Chongqing, People's Republic of China

\section{Introduction}

Olfactory disorders are common with about one-fifth of the general population exhibiting decreased olfactory acuity and approximately $5 \%$ showing anosmia [1]. Olfactory dysfunction also can be seen as an early sign of neurodegenerative diseases such as Alzheimer's or Parkinson's disease [2]. Hence, detection of olfactory dysfunction receives an increasing amount of interest.

Overall, psychophysical tests including odor detection threshold, odor discrimination, and odor identification are widely distributed [3] While they are of high significance in the clinical routine, many factors may affect the reliability and repeatability of the test, such as culture [4] and education [5] Electrophysiological tests of olfactory function are available but are typically restricted to highly specialized centers [1] 
Functional magnetic resonance imaging (fMRI) offers a noninvasive approach to assess olfactory function in an "objective" way [6]. On the cortical level, odor information is first processed in the primary olfactory areas (POC) including the piriform cortex, entorhinal cortex and amygdala. Further, olfactory information is processed in the secondary olfactory areas including the orbitofrontal cortex (OFC), insula, anterior cingulate cortex (ACC), striatal areas, thalamus, superior temporal gyrus, and hippocampus [7-9]. Previous studies have confirmed on a group level that subjects with olfactory dysfunction had reduced brain activation to odor stimuli in POC, OFC, and insular cortex compared to normosmic groups with normal olfaction [10-12]. No research has been done specifically to look into olfactory fMRI on an individual level among subjects with olfactory dysfunction. Olfactory fMRI has not reached routine clinical applications in patients with olfactory dysfunction. Therefore, the present study set out to examine how well fMRI signals to odorous stimuli could discriminate between individuals with normal olfactory function and people with little or no olfactory function.

\section{Materials and methods}

\section{Participants}

Thirty-eight subjects participated (mean age 46.6 years; 21 females, males) in this study. The cohort was divided into two groups according to their olfactory function. Of those, 22 were subjects (11 females) with diagnosed olfactory dysfunction, which was confirmed followed a standardized method [13] including standardized psychophysical testing of olfactory threshold, identification, and discrimination with the "Sniffin' Sticks" test battery [14] patients' characteristics were listed in Table 1. The rest of the participants consisted of 16 normosmic subjects (10 females). A detailed medical history was taken including conditions that potentially may have affected olfaction such as significant head trauma, chronic rhino sinusitis, neurological/endocrinological disorders, and previous nasal surgery. All included participants were in good health. Prior to inclusion in the study they received an otorhinolaryngological examination including nasal endoscopy. All participants were informed of the testing procedures and signed written informed consent before the experiment. The study design was in accordance with the Declaration of Helsinki and had been approved by the Ethics Committee of the Medical Faculty Carl Gustav Carus at the Technical University of Dresden.
Table 1 Patients' characteristics

\begin{tabular}{|c|c|c|c|c|c|}
\hline & No & $\begin{array}{l}\text { Overall } \\
\text { olfactory } \\
\text { function } \\
\text { (TDI score) }\end{array}$ & $\begin{array}{l}\text { Duration of } \\
\text { olfactory loss } \\
\text { [months] }\end{array}$ & $\begin{array}{l}\text { Age } \\
\text { [years] }\end{array}$ & Sex \\
\hline \multirow{14}{*}{$\begin{array}{l}\text { Congenital } \\
\text { olfac- } \\
\text { tory loss } \\
(n=14)\end{array}$} & P01 & 13 & & 67 & Female \\
\hline & P02 & 11 & & 64 & Male \\
\hline & $\mathrm{P} 03$ & 12.5 & & 48 & Female \\
\hline & P04 & 14 & & 23 & Male \\
\hline & P05 & 17.5 & & 19 & Male \\
\hline & P06 & 12 & & 34 & Male \\
\hline & P07 & 11 & & 27 & Female \\
\hline & P08 & 7.25 & & 30 & Male \\
\hline & P09 & 13 & & 30 & Female \\
\hline & P10 & 16 & & 39 & Female \\
\hline & $\mathrm{P} 11$ & 12 & & 23 & Male \\
\hline & P12 & 10 & & 76 & Female \\
\hline & P13 & 9 & & 22 & Male \\
\hline & P14 & 15.25 & & 22 & Female \\
\hline \multirow{8}{*}{$\begin{array}{l}\text { Idiopathic } \\
\text { olfactory } \\
\text { dysfunc- } \\
\text { tion }(n=8)\end{array}$} & P15 & 10 & 9 & 33 & Female \\
\hline & P16 & 18.25 & 12 & 64 & Female \\
\hline & P17 & 14 & 108 & 57 & Male \\
\hline & P18 & 11 & 48 & 54 & Female \\
\hline & P19 & 6.25 & 48 & 70 & Male \\
\hline & $\mathrm{P} 20$ & 1 & 96 & 55 & Female \\
\hline & $\mathrm{P} 21$ & 18 & 36 & 58 & Male \\
\hline & P22 & 9 & 84 & 60 & Male \\
\hline
\end{tabular}

\section{Assessment of olfactory function}

All participants received birhinal olfactory testing using the validated and reliable "Sniffin' Sticks" battery (Burghart, Wedel, Germany). The test consists of measurements of odor sensitivity (phenyl ethyl alcohol odor detection thresholds), odor discrimination, and odor identification [14] First, odor threshold $(T)$ is assessed using phenyl ethyl alcohol (a roselike odor) presented by means of a single staircase, using stepwise dilutions in a row of 16 felt tip pens. Second, odor discrimination $(D)$ is assessed by asking subjects to perform a three-alternative forced choice task using 16 pairs of odors. Third, odor identification $(I)$ is assessed by asking subjects to identify 16 individual odors from a list of four verbal descriptors using a forced choice task. Scores from threshold $(T)$, discrimination $(D)$ and identification $(I)$ were then added up to provide the TDI score The TDI score was the base for the diagnosis of olfactory dysfunction as well as its severity (e.g. hyposmia or anosmia). The TDI score ranges from 1 to 48 , with a score above 30.5 being considered as normosmia, TDI score between 16 and 30.5 as hyposmia, and TDI score less than 16 as functional anosmia $[15,16]$ 


\section{Odor stimulation paradigm}

One odor was used as stimuli: "Coffee" odor (Frey und Lau, Henstedt-Ulzburg, Germany). Odorless air served as control stimulus. A block design was adopted for odor stimulation during fMRI measurements. During each functional run, each block lasted for $20 \mathrm{~s}$, during one block, odorized air and was delivered to the participants' bilateral nostril intermittently ( $8 \mathrm{~s}$ for odorized air, followed by $12 \mathrm{~s}$ of odorless air), in order to minimize adaptation to the odors which refers to a recent literature indicating larger BOLD signal in response to odor stimulation in shorter runs compared to longer but fewer runs [17], totally 12 blocks and lasting $240 \mathrm{~s}$. Odorized and odorless air were delivered at a flow of $21 / \mathrm{min}$ using a portable olfactometer.

\section{MRI data acquisition and preprocessing}

A 3 T MRI scanner (Siemens Prisma, Erlangen, Germany) with a 32-channel head coil was used for image acquisition. For functional image collection, a spin echo/echo planar imaging (EPI) sequence was applied with echo time $(\mathrm{TE})=30 \mathrm{~ms}$, repetition time $(\mathrm{TR})=2000 \mathrm{~ms}$, flip angle $=90^{\circ}$, and in-plan resolution $2 \mathrm{~mm}$. A T1-weighted structural scan was collected after functional runs with 121 slices, voxel size $1 \times 1 \times 1 \mathrm{~mm}^{3}$.

Imaging data were analyzed by means of SPM12 (Wellcome Trust Centre for Neuroimaging, London, UK) implemented in Matlab (version 2013a, Mathworks, Natick, MA, USA). Functional image volumes were pre-processed, starting with realignment and unwarp. In addition, the high-resolution T1 image was co-registered to the mean image of the EPI series for each participant. Co-registered T1-weighted MR images were segmented for gray and white matter to compute spatial transformation parameters for normalization. The registered functional scans were normalized to a standard Montreal Neurological Institute (MNI) template. Normalized images were spatially smoothed with an 8-mm full-width half-maximum Gaussian kernel. Finally, removal of head motion artifacts using ArtRepair (version 4, Stanford University) was applied to the preprocessed images based on the following rules: image to image motion less than $0.5 \mathrm{~mm} / \mathrm{TR}$ and total images repaired less than $20 \%$.

\section{MRI data analysis}

First, MRI scans were checked by an experienced radiologist for possible pathological brain anomalies. On the first level, the contrast "odor ON vs odor OFF" was modeled for each participant, in each block, we used the first $8 \mathrm{~s}$ coffee odor stimulation compared to the last $8 \mathrm{~s}$ odorless air (Fig. 1). The 1 st level contrasts were submitted into second level analysis to show: (1) brain activation in patients and normosmic

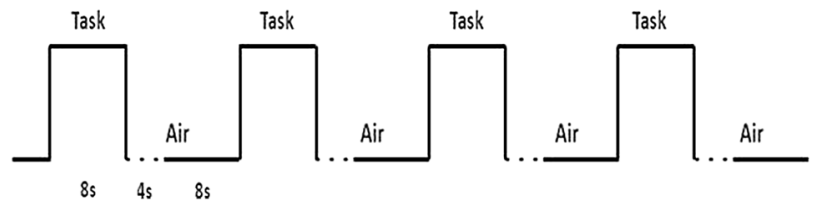

Fig. 1 fMRI block design paradigm, totally 12 times of repeated "Task-Air" cycles. The time for each task is $8 \mathrm{~s}$, the total time for presenting air is $12 \mathrm{~s}$, during the odorless presentation, the first $4 \mathrm{~s}$ are buffer time

groups separately, and (2) different brain activation between the two groups. Analyses were focused on three regions of interest (ROIs): the primary olfactory cortex (POC), the olfactory orbitofrontal cortex and the insular cortex. The anatomical mask defined by Fjaeldstad et al.[8] including the piriform cortex and part of the amygdala, was used as the mask for POC. The olfactory OFC mask was built based on a 10 -mm sphere centered on the right $(x, y, z: 24,36,-12)$ or left putative olfactory OFC $(x, y, z:-22,32,-16)$ [17]. The insular cortex ROI was defined with the WFU_PickAtlas software (ANSIR, Wake Forest University, Winston-Salem, NC, USA)[18] . The template of the region for each ROI was showed in Fig. 2.

On an individual level, we conducted anatomical regionof-interest (ROI) analyses by extracting the mean \% BOLD signal change (\% BOLD signal change is often calculated using a baseline of the mean of the time series on a voxel, the MarsBaR software will calculate a baseline from the mean signal over a small ROI of many voxels) for each participant from all voxels in three ROIs [19] The anatomical ROI approach increases statistical power by averaging BOLD activation across multiple voxels within a specific brain region using the MarsBaR toolbox [20]. thereby reducing noise and the need to correct for multiple comparisons. Critically, anatomical ROIs are independent of the functional data analyzed, resulting in unbiased estimates of effect size [21].

Next, the spatial distribution of activation extent (defined as the number of significantly activated voxels) and intensity (defined as the cluster peak $z$ value) were also extracted for individual participants from each ROI. An activation was considered significant when surviving to an uncorrected threshold of $p<0.05$. The overall visualization and screenshots were performed using the software of MRIcroGL (https://www.cabiatl.com/mricr ogl/). The receiver operating characteristic (ROC) curve was constructed to test the ability of the \% BOLD signal change within each of the three ROIs to discriminate between people with normal olfaction and patient with olfactory dysfunctions, defined based on their TDI scores. The classification accuracy was quantified using the area under the (AUC) value. All statistical analysis was 
Fig. 2 The light blue regions stand for the selected ROIs template: POC, OFC and Insular

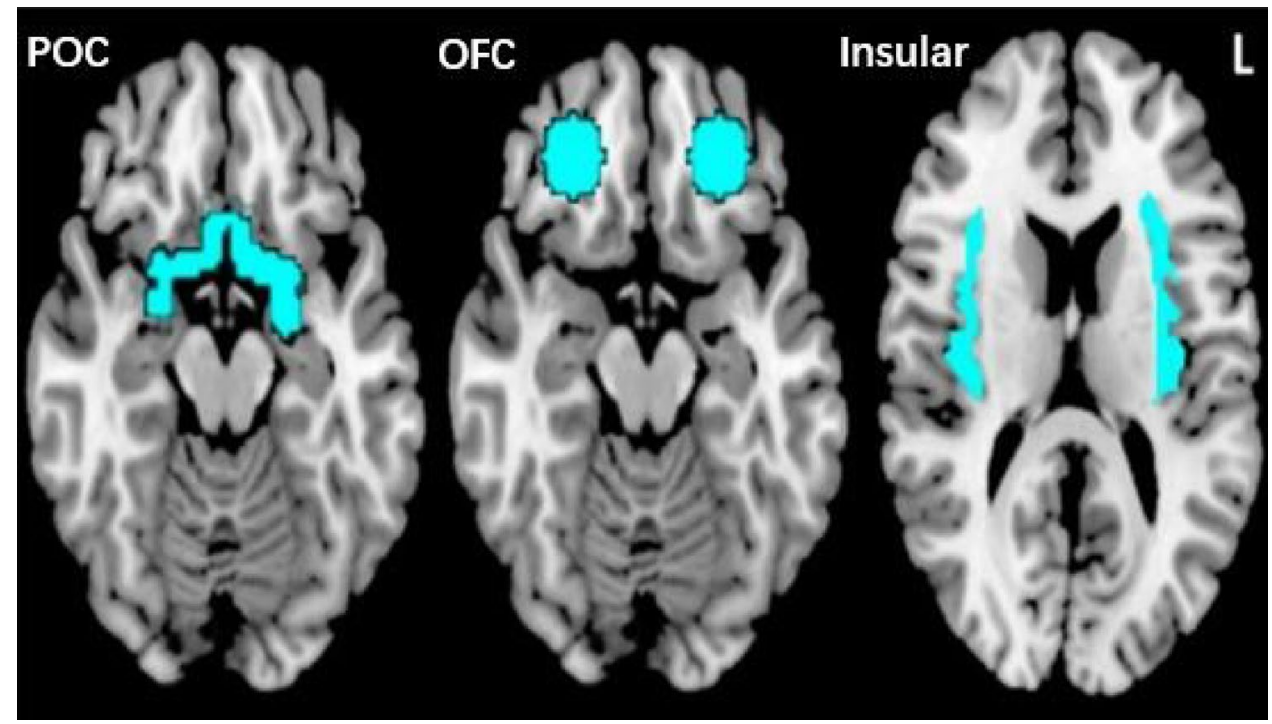

performed by means of SPSS 23 (SPSS Inc., Chicago, IL, USA). Pearson correlation was performed between activations response of odor stimulation of selected ROIs of individuals and the Sniffin' Sticks test scores. Chi-square analysis was used in calculating the proportion of effective activations response of odor stimulation of selected ROIs of individuals between normosmics and subjects with olfactory dysfunction.

\section{Results}

\section{Group level brain activation}

Brain activations to odor stimulation in the normosmic group were shown in Fig. 3a, the local maxima of the main activated areas were found in the left POC (Montreal Neurological Institute [MNI] peak coordinates $-26,-2,-18$ ), insula (MNI peak coordinates $-38,-8$, 6 and $36,0,16)$, no obvious activations were found in the OFC (Fig. 3a). In subjects, the local maxima of the main activated areas were found in the left insula (MNI peak coordinates $-36,-2,18)$, no obvious activations were found in the POC and OFC regions (Fig. 3b). Between group comparison revealed stronger activation in the POC among normosmics as compared to subjects. Table 2 recaps maximum activation voxels and peak- $z$ value responses to olfactory stimulation obtained from the the POC, OFC and insula regions in the group-level and found that the intensity of activation response to odor stimulation was much higher in the insular region than other ROIs.

\section{Individual variability}

Tables 3 and 4 show the $\%$ of BOLD signal change, cluster size (assessment of significant activation in fMRI) and peak$z$ value (normalized magnitude of peak voxel from statistical parametric map from publication) in responses to odor stimulation obtained from the three ROIs ROI for each participant. No significant correlation between TDI scores and the $\%$ of BOLD signal change, cluster size or peak- $z$ value was found $(p>0.05)$.

Table 4 summarizes the cluster size and peak $z$-value of each ROIs for each participant. With the applied threshold (uncorrected $p<0.05$ ), 93.8\%, 62.5\%, 62.5\% of the normosmics showed detectable cluster (minimum 1 voxel) in the POC, OFC or insula, respectively. In the dysosmic group, the percentage of participants with observed activated clusters was $40.9 \%, 59.1 \%$ and $68.2 \%$ for the POC, OFC and insula, respectively. The proportion of activated clusters in POC in normosmic participants $(93.8 \%)$ was significantly higher than that in subjects with olfactory dysfunction $(40.9 \%)$ $\left(X^{2}=14.9, P<0.001\right)$ ROC analyses showed no significant AUC for $\%$ BOLD signal change for all three ROIs (POC: $\mathrm{AUC}=0.53, p=0.72$; OFC: $\mathrm{AUC}=0.58, p=0.41$; insula: $\mathrm{AUC}=0.67, p=0.07)$, meaning that it was not possible to distinguish between normosmics and subjects with olfactory dysfunction by means of the \% BOLD signal change.

\section{Discussion}

The present study explored the odor-induced brain activation at an individual level among subjects with olfactory dysfunction and normosmics with a normal sense of smell. 


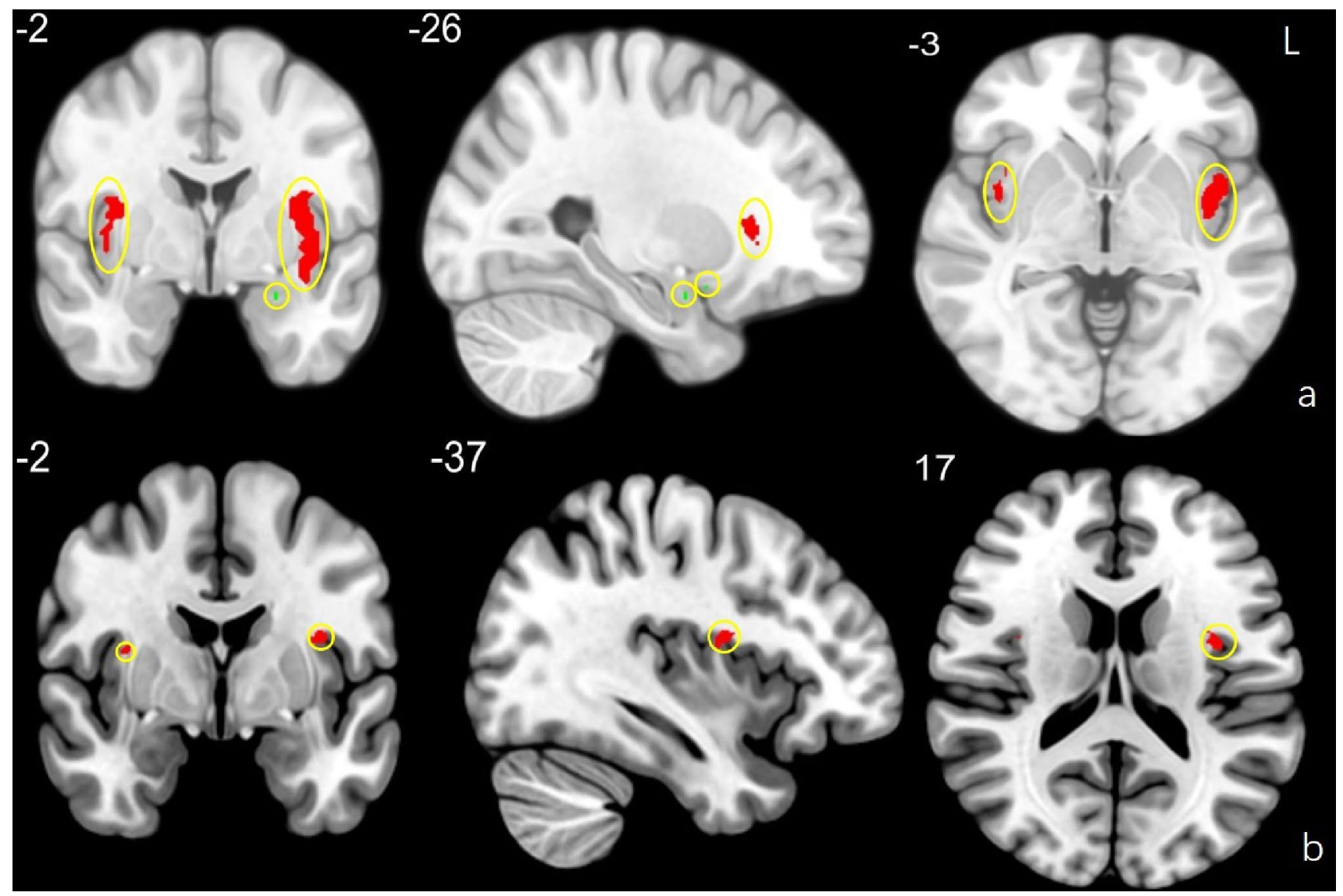

Fig. 3 (a) fMRI in 3 dimensions from a normosmic subject, red region indicates the activated voxel clusters in the insular, green region indicates the activated voxel clusters in the POC. (b) fMRI in
3 dimensions from a subject with olfactory dysfunction, red region indicates the activated voxel clusters in the insular. Yellow circle is used to emphasize the activated voxel clusters
Results suggest a large inter-individual variability, including the average BOLD signal in predicted ROIs, peak $z$ value as well as the activated cluster size at a given threshold. While the between-group comparison revealed stronger activation among normosmics than subjects with olfactory dysfunction, subjects with olfactory loss could not be separated from normosmics according to their BOLD responses based on individual information. In other words, the BOLD signal on an individual level could not predict olfactory performance accurately, though the present study indicated that there is a significantly higher proportion of odor-induced activations in the POC of normosmics compared to subjects with olfactory dysfunction. Previous research has indicated high variability of olfactory fMRI among normosmics [22] . Our present results corroborate and extend these findings showing the variability in subjects with olfactory dysfunction.

The olfactory BOLD signal suffers from the magnetic artifact which decreases the signal-to-noise ratio (SNR), which in turn lowers the sensitivity to detect the BOLD signal in the olfactory system. Besides, physiological factors such as respiration and metabolic status also influence the sensitivity for detecting differences between groups. Some approaches have been suggested to increase SNR regarding the odor stimulation paradigm, e.g., short odor stimulation time $[23,24]$ rapid repetition time $(<1 \mathrm{~s})$ during brain image acquisition [25] or task protocols applied (e.g. synchronization of the breathing cycle with odor stimulation) [23] In addition, sequential stimulation with increasing intensities of odor may potentially offset potential habituation effects [26] Moreover, the presently used odors were familiar foodrelated. This association to food may constitute an angle through which the odor could be influenced by affective or metabolic factors. Unfamiliar neutral odors (e.g. "olfactory white" [27] may be a possible alternative choice.

The challenge of developing fMRI biomarkers for individual subjects is a general issue, due to the relatively low reliability of the fMRI signal [28,29]. As stated above, the individual difference measured with fMRI may also be attributed to the noise from the measurement [30]. Further, within-subject variance such as motion and physiological changes may also influence the stability of the measurement which additionally influences the weak olfactory signal with 
Table 2 Data statistics for individuals, BOLD signal change obtained in POC, OFC, insular from each subject in response to odor stimulation

\begin{tabular}{|c|c|c|c|c|c|c|c|}
\hline & & \multirow[t]{2}{*}{ TDI } & \multirow[t]{2}{*}{ Age (years) } & \multirow[t]{2}{*}{ Sex } & \multicolumn{3}{|c|}{ ROI (signal change\%) } \\
\hline & & & & & POC & OFC & Insular \\
\hline \multirow{22}{*}{$\begin{array}{r}\text { Subject with olfactory } \\
\text { dysfunction }(n=22)\end{array}$} & P01 & 13.00 & 67 & $\mathrm{f}$ & 0.08 & 0.05 & 0.03 \\
\hline & $\mathrm{P} 02$ & 11.00 & 64 & $\mathrm{~m}$ & 0.04 & 0.11 & 0.00 \\
\hline & P03 & 12.50 & 48 & $\mathrm{~m}$ & 0.06 & -0.05 & 0.01 \\
\hline & $\mathrm{P} 04$ & 14.00 & 23 & $\mathrm{~m}$ & -0.20 & -0.11 & -0.09 \\
\hline & P05 & 17.50 & 19 & $\mathrm{~m}$ & 0.10 & -0.05 & -0.02 \\
\hline & P06 & 12.00 & 34 & $\mathrm{~m}$ & -0.05 & -0.04 & -0.04 \\
\hline & P07 & 11.00 & 27 & $\mathrm{f}$ & 0.17 & 0.10 & 0.29 \\
\hline & P08 & 7.25 & 30 & $\mathrm{~m}$ & 0.02 & 0.08 & -0.07 \\
\hline & P09 & 13.00 & 30 & $\mathrm{f}$ & 0.14 & 0.30 & -0.01 \\
\hline & $\mathrm{P} 10$ & 10.00 & 33 & $\mathrm{f}$ & 0.00 & -0.02 & -0.16 \\
\hline & $\mathrm{P} 11$ & 16.00 & 39 & $\mathrm{f}$ & -0.05 & 0.20 & 0.56 \\
\hline & $\mathrm{P} 12$ & 18.25 & 64 & $\mathrm{f}$ & 0.00 & -0.01 & -0.07 \\
\hline & $\mathrm{P} 13$ & 14.00 & 57 & $\mathrm{~m}$ & 0.11 & 0.13 & -0.06 \\
\hline & P14 & 12.00 & 23 & $\mathrm{~m}$ & -0.26 & -0.16 & -0.28 \\
\hline & P15 & 11.00 & 54 & $\mathrm{f}$ & -0.02 & -0.06 & -0.05 \\
\hline & P16 & 6.25 & 70 & $\mathrm{~m}$ & -0.21 & -0.06 & -0.25 \\
\hline & P17 & 1.00 & 55 & $\mathrm{f}$ & 0.01 & 0.11 & 0.20 \\
\hline & P18 & 10.00 & 76 & $\mathrm{f}$ & 0.07 & -0.05 & -0.17 \\
\hline & P19 & 18.00 & 58 & $\mathrm{~m}$ & -0.03 & 0.09 & 0.03 \\
\hline & $\mathrm{P} 20$ & 9.00 & 22 & $\mathrm{~m}$ & -0.11 & 0.02 & -0.09 \\
\hline & $\mathrm{P} 21$ & 15.25 & 22 & $\mathrm{~m}$ & -0.16 & -0.03 & 0.02 \\
\hline & $\mathrm{P} 22$ & 9.00 & 60 & $\mathrm{~m}$ & -0.04 & 0.05 & -0.03 \\
\hline \multirow{16}{*}{$\begin{array}{l}\text { Normosmia } \\
(n=16)\end{array}$} & $\mathrm{C} 01$ & 30.75 & 59 & $\mathrm{f}$ & 0.14 & 0.14 & 0.07 \\
\hline & $\mathrm{C} 02$ & 30.25 & 65 & $\mathrm{~m}$ & 0.03 & 0.12 & -0.01 \\
\hline & $\mathrm{C} 03$ & 34.00 & 48 & $\mathrm{f}$ & -0.14 & 0.07 & 0.05 \\
\hline & $\mathrm{C} 04$ & 39.50 & 45 & $\mathrm{~m}$ & 0.23 & 0.11 & 0.11 \\
\hline & $\mathrm{C} 05$ & 38.00 & 49 & $\mathrm{f}$ & -0.04 & -0.01 & 0.12 \\
\hline & C06 & 30.00 & 48 & $\mathrm{f}$ & 0.07 & 0.01 & 0.03 \\
\hline & C07 & 36.00 & 30 & $\mathrm{f}$ & -0.06 & -0.06 & -0.05 \\
\hline & $\mathrm{C} 08$ & 31.00 & 59 & $\mathrm{f}$ & -0.08 & 0.04 & 0.07 \\
\hline & C09 & 39.50 & 57 & $\mathrm{f}$ & 0.04 & 0.12 & 0.02 \\
\hline & $\mathrm{C} 10$ & 35.50 & 37 & $\mathrm{f}$ & 0.12 & -0.05 & -0.03 \\
\hline & C11 & 38.50 & 64 & $\mathrm{f}$ & -0.02 & -0.09 & -0.12 \\
\hline & $\mathrm{C} 12$ & 34.50 & 21 & $\mathrm{~m}$ & 0.16 & 0.28 & 0.48 \\
\hline & C13 & 40.50 & 48 & $\mathrm{~m}$ & -0.13 & -0.14 & -0.17 \\
\hline & $\mathrm{C} 14$ & 37.25 & 43 & $\mathrm{f}$ & -0.08 & 0.13 & 0.06 \\
\hline & $\mathrm{C} 15$ & 30.75 & 55 & $\mathrm{~m}$ & 0.04 & 0.24 & 0.12 \\
\hline & C16 & 37.75 & 59 & $\mathrm{~m}$ & -0.06 & -0.04 & -0.07 \\
\hline
\end{tabular}

low signal-to-noise ratio. It has been suggested that precision functional mapping of individual human brains can be achieved with larger datasets [31].

One recent study demonstrated that odor-induced brain activation in the amygdala covaried with the participants' odor sensitivity [23]. However, such correlation does not ensure the generalizability of the established relationship to out-of-sample individual subjects. Future research should aim to shift from such simple correlations to predictions. We may be able to interpret the fMRI-derived statistics of an individual subject in light of its distribution in a larger, normative sample. This approach could lead, for clinical imaging, to a more biologically informed science of human disease and a better basis for personalized treatment. Besides, with the advances in understanding the precise functional regions for olfactory processing and the higher resolution imaging technique, more precise location for the brain structures involved in initial processing of odor stimuli could be identified. 
Table 3 Cluster size, peak- $z$ value obtained in POC, OFC, Insular from each subject in response to odor stimulation

\begin{tabular}{|c|c|c|c|c|c|c|c|}
\hline & & \multicolumn{2}{|l|}{ POC } & \multicolumn{2}{|l|}{ OFC } & \multicolumn{2}{|l|}{ Insular } \\
\hline & & $\begin{array}{l}\text { Cluster size } \\
\text { (voxel) }\end{array}$ & $\begin{array}{l}\text { Peak- } z \\
\text { score }\end{array}$ & $\begin{array}{l}\text { Cluster size } \\
\text { (voxel) }\end{array}$ & $\begin{array}{l}\text { Peak- } z \\
\text { score }\end{array}$ & $\begin{array}{l}\text { Cluster size } \\
\text { (voxel) }\end{array}$ & $\begin{array}{l}\text { Peak-z } \\
\text { score }\end{array}$ \\
\hline \multirow{22}{*}{$\begin{array}{l}\text { Subject with olfac- } \\
\text { tory dysfunction } \\
(n=22)\end{array}$} & P01 & 5 & 1.65 & 20 & 2.04 & 9 & 2.17 \\
\hline & P02 & 7 & 2.04 & 38 & 2.18 & 1 & 1.73 \\
\hline & P03 & & -4.00 & - & - & - & - \\
\hline & P04 & - & - & 3 & 1.80 & - & - \\
\hline & P05 & - & - & - & - & 3 & 1.72 \\
\hline & P06 & 18 & 1.69 & 31 & 2.22 & 14 & 2.11 \\
\hline & P07 & - & - & 8 & 1.94 & 46 & 2.40 \\
\hline & P08 & - & - & 10 & 2.39 & 123 & 2.85 \\
\hline & P09 & 59 & 2.22 & 104 & 2.92 & 128 & 3.60 \\
\hline & P10 & - & - & 21 & 2.33 & 138 & 2.44 \\
\hline & P11 & - & - & 6 & 2.14 & - & - \\
\hline & P12 & - & - & 1 & 1.64 & 12 & 1.97 \\
\hline & $\mathrm{P} 13$ & - & - & - & - & - & - \\
\hline & P14 & - & - & - & - & - & - \\
\hline & P15 & - & - & - & - & - & - \\
\hline & P16 & 12 & 1.99 & 20 & 2.69 & 59 & 2.13 \\
\hline & P17 & 23 & 2.28 & 7 & 1.85 & 90 & 2.63 \\
\hline & P18 & - & - & - & - & 1 & 1.75 \\
\hline & P19 & 2 & 1.41 & - & - & - & - \\
\hline & $\mathrm{P} 20$ & 19 & 1.93 & - & - & 1 & 1.66 \\
\hline & $\mathrm{P} 21$ & - & - & 14 & 2.26 & 12 & 1.89 \\
\hline & $\mathrm{P} 22$ & - & - & - & - & 18 & 2.22 \\
\hline \multirow{16}{*}{$\begin{array}{l}\text { Normosmia } \\
(n=16)\end{array}$} & $\mathrm{C} 01$ & 1 & 0.01 & 1 & 1.89 & 4 & 1.82 \\
\hline & $\mathrm{C} 02$ & 16 & 1.85 & 27 & 2.14 & 36 & 2.26 \\
\hline & $\mathrm{C} 03$ & 8 & 1.84 & - & - & 4 & 2.20 \\
\hline & $\mathrm{C} 04$ & - & - & - & - & - & - \\
\hline & $\mathrm{C} 05$ & 114 & 3.16 & 164 & 3.25 & 1503 & 5.57 \\
\hline & C06 & 1 & 1.31 & - & - & - & - \\
\hline & $\mathrm{C} 07$ & 7 & 1.84 & - & - & - & - \\
\hline & C08 & 5 & 1.55 & 38 & 2.65 & 1 & 1.81 \\
\hline & C09 & 5 & 1.56 & - & - & - & - \\
\hline & C10 & 4 & 1.59 & - & - & 13 & 2.11 \\
\hline & C11 & 3 & 1.42 & 1 & 1.79 & 2 & 1.87 \\
\hline & $\mathrm{C} 12$ & 112 & 4.52 & 231 & 3.44 & 1443 & 5.12 \\
\hline & C13 & 2 & 1.43 & 31 & 2.46 & - & - \\
\hline & C14 & 2 & 1.41 & 72 & 2.65 & 3 & 1.98 \\
\hline & C15 & 1 & 1.35 & 60 & 2.54 & 473 & 3.76 \\
\hline & C16 & 12 & 2.34 & 10 & & - & - \\
\hline
\end{tabular}

-means no obvious activations obtained in this ROI

\section{Conclusion}

The present study showed poor separation between individual normosmics and individual subjects with olfactory dysfunction based on odor-induced activation in key olfactory brain regions. Across the two groups the $\mathrm{POC}$ region showed more activation to odors in normosmic subjects compared to subjects with olfactory dysfunction. At present it appears problematic to diagnose olfactory dysfunction on an individual level using fMRI. 
Table 4 Cluster size, peak- $z$ value in response to odor stimulation obtained from POC, OFC and Insula ROIs in normosmic subjects, subjects with olfactory dysfunction and normosmic group against subjects with olfactory dysfunction

\begin{tabular}{lllllll}
\hline & $k$ & $z$ & $x$ & $y$ & $z$ & ROIs \\
\hline Normosmics & 2 & 2.76 & -26 & -2 & -18 & POC \\
& - & - & - & - & - & OFC \\
Subjects with olfactory dysfunction & - & - & - & - & - & Insula \\
& - & - & - & - & - & OFC \\
Normosmics > subject with olfactory & 16 & 2 & -36 & -2 & 18 & Insula \\
dysfunction & 3 & 2.12 & -26 & 8 & -16 & POC \\
& - & - & - & - & - & OFC \\
& 201 & 2.65 & -36 & -6 & 4 & Insula \\
\hline
\end{tabular}

-means no obvious activations obtained in this ROI.
Funding Open Access funding provided by Projekt DEAL.

\section{Compliance with ethical standards}

Conflict of interest This research received financial support by the Deutsche Forschungsgemeinschaft to TH (DFG HU411/18-1). All other authors declare that they have no conflict of interest.

Research involving human participants All procedures performed in studies involving human participants were in accordance with the ethical standards of the institutional and/or national research committee and with the 1964 Helsinki Declaration and its later amendments or comparable ethical standards. The study was approved by the Ethics committee of the Medical Faculty of the Technical University of Dresden (EK441102016). This article does not contain any studies with animals performed by any of the authors.

Informed consent Informed consent was obtained from all individual participants included in the study.

Open Access This article is licensed under a Creative Commons Attribution 4.0 International License, which permits use, sharing, adaptation, distribution and reproduction in any medium or format, as long as you give appropriate credit to the original author(s) and the source, provide a link to the Creative Commons licence, and indicate if changes were made. The images or other third party material in this article are included in the article's Creative Commons licence, unless indicated otherwise in a credit line to the material. If material is not included in the article's Creative Commons licence and your intended use is not permitted by statutory regulation or exceeds the permitted use, you will need to obtain permission directly from the copyright holder. To view a copy of this licence, visit http://creativecommons.org/licenses/by/4.0/.

\section{References}

1. Hummel T, Whitcroft KL, Andrews P et al (2020) Position paper on olfactory dysfunction. Rhinology 56(1):1-30

2. Wilson RS, Arnold SE, Schneider JA, Boyle PA, Buchman AS, Bennett DA (2009) Olfactory impairment in presymptomatic Alzheimer's disease. Ann N Y Acad Sci 10:730-735
3. Doty RL (2015) Olfactory dysfunction and its measurement in the clinic. World J Otorhinolaryngol Head Neck Surg 1(1):28-33

4. Ribeiro JC, Simoes J, Silva F et al (2016) Cultural adaptation of the portuguese version of the "sniffin' sticks" smell test: reliability, validity, and normative data. PLoS ONE 11(2):e0148937

5. Orhan KS, Karabulut B, Keles N, Deger K (2012) Evaluation of factors concerning the olfaction using the Sniffin' Sticks test. Otolaryngol Head Neck Surg 146(2):240-246

6. Howard JD, Plailly J, Grueschow M, Haynes JD, Gottfried JA (2009) Odor quality coding and categorization in human posterior piriform cortex. Nat Neurosci 12(7):932-938

7. Seubert J, Freiherr J, Djordjevic J, Lundstrom JN (2013) Statistical localization of human olfactory cortex. Neuroimage 66:333-342

8. Fjaeldstad A, Fernandes HM, Van Hartevelt TJ et al (2020) Brain fingerprints of olfaction: a novel structural method for assessing olfactory cortical networks in health and disease. Sci Rep 7:42534

9. Lundstrom JN, Boesveldt S, Albrecht J (2011) Central processing of the chemical senses: an overview. ACS chemical NeuroScience 2(1):5-16

10. Pellegrino R, Hahner A, Bojanowski V, Hummel C, Gerber J, Hummel T (2016) Olfactory function in patients with hyposmia compared to healthy subjects - an fMRI study. Rhinology 54(4):374-381

11. Levy LM, Henkin RI, Hutter A, Lin CS, Schellinger D (1998) Mapping brain activation to odorants in patients with smell loss by functional MRI. J Comput Assist Tomogr 22(1):96-103

12. Han P, Winkler N, Hummel C, Hahner A, Gerber J, Hummel T (2018) Impaired brain response to odors in patients with varied severity of olfactory loss after traumatic brain injury. J Neurol 265(10):2322-2332

13. Huart C, Rombaux P, Hummel T (2013) Plasticity of the human olfactory system: the olfactory bulb. Molecules 18(9):11586-11600

14. Hummel T (1997) 'Sniffin'sticks' olfactory performance assessed by the combined testing of odor identification, odor discrimination and olfactory threshold. Chem Senses. 22(1):39-52

15. Hummel T, Kobal G, Gudziol H, Mackay-Sim A (2007) Normative data for the "Sniffin' Sticks" including tests of odor identification, odor discrimination, and olfactory thresholds: an upgrade based on a group of more than 3000 subjects. Eur Arch Otorhinolaryngol 264(3):237-243

16. Kobal G, Klimek L, Wolfensberger M et al (2000) Multicenter investigation of 1036 subjects using a standardized method for 
the assessment of olfactory function combining tests of odor identification, odor discrimination, and olfactory thresholds. Eur Arch Oto-Rhino-Laryngol 257(4):205-211

17 Gottfried JA, Zald DH (2005) On the scent of human olfactory orbitofrontal cortex: meta-analysis and comparison to nonhuman primates. Brain Res Rev 50(2):287-304

18. Maldjian JA, Laurienti PJ, Kraft RA, Burdette JH (2003) An automated method for neuroanatomic and cytoarchitectonic atlas-based interrogation of fMRI data sets. NeuroImage 19(3):1233-1239

19. Lawrence NS, Hinton EC, Parkinson JA, Lawrence AD (2012) Nucleus accumbens response to food cues predicts subsequent snack consumption in women and increased body mass index in those with reduced self-control. NeuroImage 63(1):415-422

20. Brett M, Anton J-L, Valabregue R, Poline J-B (2002) Region of interest analysis using an SPM toolbox paper presented at: 8th international conference on functional mapping of the human brain. NeuroImage 16:2

21. Kriegeskorte N, Simmons WK, Bellgowan PS, Baker CI (2009) Circular analysis in systems neuroscience: the dangers of double dipping. Nat Neurosci 12(5):535-540

22. Morrot G, Bonny JM, Lehallier B, Zanca M (2013) FMRI of human olfaction at the individual level: interindividual variability. J Magn Reson Imaging 37(1):92-100

23. Kleinhans NM, Reilly M, Blake M et al (2019) FMRI correlates of olfactory processing in typically-developing school-aged children. Psychiatry Res Neuroimaging 283:67-76

24. Han P, Zang Y, Hummel C, Faria V, Hummel T (2020) Short or long runs: an exploratory study of odor-induced fMRI design. Laryngoscope 130(5):1110-1115
25. Georgiopoulos C, Witt ST, Haller S et al (2018) Olfactory fMRI: implications of stimulation length and repetition time. Chem Senses 43(6):389-398

26. Karunanayaka P, Eslinger PJ, Wang JL et al (2014) Networks involved in olfaction and their dynamics using independent component analysis and unified structural equation modeling. Hum Brain Mapp 35(5):2055-2072

27. Weiss T, Snitz K, Yablonka A et al (2012) Perceptual convergence of multi-component mixtures in olfaction implies an olfactory white. Proc Natl Acad Sci U S A 109(49):19959-19964

28. Holiga $\breve{S}$, Sambataro F, Luzy C et al (2018) Test-retest reliability of task-based and resting-state blood oxygen level dependence and cerebral blood flow measures. NeuroImage 13(11):e0206583

29. Fröhner JH, Teckentrup V, Smolka MN, Kroemer NBJN (2019) Addressing the reliability fallacy in fMRI: similar group effects may arise from unreliable individual effects. NeuroImage 195:4-189

30. Dubois J, Adolphs R (2016) Building a science of individual differences from fmri. Trends Cogn Sci 20(6):425-443

31. Gordon EM, Laumann TO, Gilmore AW et al (2020) Precision functional mapping of individual human brains. Neuron 95(4):791-807

Publisher's Note Springer Nature remains neutral with regard to jurisdictional claims in published maps and institutional affiliations. 